\title{
Capsule Commentary on Goldman et al., Comparative Effectiveness of Multifaceted Outreach to Initiate Colorectal Cancer Screening in Community Health Centers: A Randomized Controlled Trial
}

\author{
Sharon K. Hull, MD, MPH, FACPM, FAAFP \\ Department of Community and Family Medicine, Division Chief, Family Medicine, Duke University School of Medicine, Durham, NC, USA.
}

J Gen Intern Med 30(8):1191

DOI: $10.1007 / \mathrm{s} 11606-015-3334-2$

() Society of General Internal Medicine 2015

$\mathrm{T}$ he authors conducted a randomized trial of a multidimensional intervention to enhance colorectal cancer (CRC) screening uptake using fecal occult blood tests (FOBT) in a community health center (CHC) setting for patients who had no evidence of prior screening, ${ }^{1}$ building on the author's previous work in which mailings sent to patients in this setting achieved higher CRC screening uptake than a multifaceted practice-level systems-based intervention. ${ }^{2}$ Intervention patients received mailed CRC screening kits, a letter from the patient's health care provider, and plain-language instructions for completing the test. The comparison group experienced usual care. Participants were followed for 12 months after study entry.

The intervention group patients were more likely to complete CRC screening with FOBT than those in the usual care group (36.7 \% vs. $14.8 \%$ ). Screening completion rates were higher among patients who visited the clinic more often and those who received text messages. Interestingly, the rate of positive FOBT test results was much lower in the intervention than the control group ( $6.5 \%$ vs. $16.1 \%$ ); the authors do not adequately discuss this difference to assess its cause.

It is important to note that the practice setting in which this study was conducted had implemented systems-based practice changes to support CRC screening prior to this study. Consequently, the rate of CRC screening had increased from $17 \%$ to $43 \%$ over a two-year period. ${ }^{1}$ Therefore, patients in the usual care group for this study may not be similar to those in practices where such system-level changes have not been implemented. In addition, patients in lower socioeconomic groups may be less likely to have landline telephones or permanent addresses, ${ }^{3,4}$ and the authors do not discuss the impact of this on their results.

Future studies could be enhanced by including discussion of the practical cost of such an intervention and consideration of cost-benefit analysis in both $\mathrm{CHC}$ and non-CHC settings.

Conflict of Interest: The author has no conflicts of interest with this article.

Corresponding Author: Sharon K. Hull, MD, MPH, FACPM, FAAFP; Department of Community and Family Medicine, Division Chief, Family MedicineDuke University School of Medicine, Durham, NC 27710,USA (e-mail: sharon.hull@duke.edu).

\section{REFERENCES}

1. Goldman SN, Brown T, Lee JY, Buchanan DR, Balsley K, Cesan A, Weil J, Garrity BH, Baker DW. Comparative Effectiveness of Multifaceted Outreach to Initiate Colorectal Cancer Screening in Community Health Centers: A Randomized Controlled Trial. J Gen Intern Med. doi: 10.1007/S11606-0153234-5.

2. Baker DW, Brown T, Buchanan DR, Weil J, Balsley K, Ranalli L, et al. Comparative effectiveness of a multifaceted intervention to improve adherence to annual colorectal cancer screening in community health centers: a randomized clinical trial. JAMA Intern Med. 2014. doi:10.1001/ jamainternmed.2014.2352.

3. North CS, Black DE. Predictors of successful tracking over time in a homeless population. Social Work Research. 2012;36(2):153-159.

4. Brick JM, Dipko S, Presser S, Tucker C, Yuan Y. Nonresponse bias in a dual frame sample of cell and landline numbers. Public Opinion Quarterly. 2006;70(5):780-793. 